EPJ Web of Conferences 21, 04005 (2012)

DOI: 10.1051/epjconf/20122104005

(c) Owned by the authors, published by EDP Sciences, 2012

\title{
Scissors Mode in Gd Nuclei
}

J. Kroll ${ }^{1, a}$, B. Baramsai ${ }^{2}$, J. A. Becker ${ }^{3}$, F. Bečvár ${ }^{1}$, T. A. Bredeweg ${ }^{4}$, A. Couture ${ }^{4}$, A. Chyzh²,3, D. Dashdorj ${ }^{2,3}$, R. C. Haight ${ }^{4}$, M. Jandel ${ }^{4}$, M. Krtička ${ }^{1}$, G. E. Mitchell ${ }^{2}$, J. M. O’Donnell ${ }^{4}$, W. Parker ${ }^{3}$, R. S. Rundberg ${ }^{4}$, J. L. Ullmann ${ }^{4}$, D. J. Vieira ${ }^{4}$, C.L. Walker ${ }^{2}$, J. B. Wilhelmy ${ }^{4}$, J. M. Wouters ${ }^{4}$, C. $\mathrm{Y} . \mathrm{Wu}^{3}$.

1 Charles University in Prague, CZ-180 00 Prague 8, Czech Republic

2 North Carolina State University, Raleigh, NC 27695 and Triangle Universities Nuclear Laboratory, Durham, NC 27708

3 Lawrence Livermore National Laboratory, Livermore, CA 94551

4 Los Alamos National Laboratory, Los Alamos, New Mexico 87545

\begin{abstract}
Spectra of $\gamma$ rays following neutron capture at isolated resonances of 6 stable Gd isotopes were measured with highly segmented $\mathrm{BaF}_{2}$ detector DANCE at the Los Alamos LANSCE spallation neutron source. The main emphasis was put on studying the $\gamma$-cascade decay of neutron resonances to get unique information on photon strength. An analysis of the accumulated $\gamma$-ray spectra within the extreme statistical model leads to an inescapable conclusion that scissors mode resonances are built not only on the groundstate, but also on excited levels in all product nuclei studied. The results on summed $B(M 1) \uparrow$ strength and energy of the scissors mode are compared with systematics of scissors mode parameters for the ground-state transitions deduced from nuclear resonance fluorescence measurements. A specific feature of our experiments is the investigation of scissors mode of odd nuclei, for which the nuclear resonance fluorescence provides only limited information.
\end{abstract}

\section{Introduction}

In medium and heavy mass nuclei detailed spectroscopic information exists only for levels at low excitation energy near the ground state and often for resonances above the neutron separation energy $S_{n}$. Due to the rapid increase of the level density with excitation energy, it is extremely difficult to resolve the populating or depopulating transitions in order to obtain reliable spectroscopic information in intermediate energy region below $S_{n}$ that is usually called a level quasicontinuum. It is believed that $\gamma$ decay of the nucleus in the quasicontinuum is described by the extreme statistical model in terms of the nuclear level density and a set of photon strength functions (PSFs) for different multipolarities. Probably the most direct way to examine these quantities is via study of the properties of $\gamma$-ray spectra originating from the radiative neutron capture reaction at isolated resonances.

The combination of the pulsed neutron beam at LANSCE (Los Alamos Neutron Science CEnter) and the highly segmented, highly efficient $\gamma$ calorimeter DANCE (Detector for Advanced Neutron Capture Experiments) provides an ideal opportunity to study these $\gamma$-ray spectra.

In this contribution we describe a measurement of the series of stable Gd isotopes using the DANCE calorimeter. A major goal of these experiments is to use $\gamma$-ray spectra measured for various multiplicities to determine the appropriate PSFs with special interest in the behavior of the scissors mode which is expected to play an important role in $\gamma$-decay of Gd nuclei.

In Sec. 2 the experimental technique to measure the $\gamma$-ray spectra is described. The modeling of the statistical $\gamma$ cascades is discussed in Sec. 3. Information about the PSFs that can be obtained from

\footnotetext{
${ }^{a}$ e-mail: kroll@ipnp.troja.mff.cuni.cz
}

This is an Open Access article distributed under the terms of the Creative Commons Attribution-Noncommercial License 3.0, which permits unrestricted use, distribution, and reproduction in any noncommercial medium, provided the original work is properly cited. 
EPJ Web of Conferences

Table 1. Isotopic composition and the average thickness of the Gd targets used in the measurements.

\begin{tabular}{|c|c|c|c|c|c|c|c|c|}
\hline \hline Target & \multicolumn{7}{|c|}{ Isotope abundance (\%) } & \\
\hline & ${ }^{152} \mathrm{Gd}$ & ${ }^{154} \mathrm{Gd}$ & ${ }^{15} \mathrm{Gd}$ & ${ }^{156} \mathrm{Gd}$ & ${ }^{157} \mathrm{Gd}$ & ${ }^{158} \mathrm{Gd}$ & ${ }^{160} \mathrm{Gd}$ & Average thickness $\left(\mathrm{mg} / \mathrm{cm}^{2}\right)$ \\
\hline${ }^{152} \mathrm{Gd}$ & 42.49 & 4.38 & 15.93 & 13.91 & 7.82 & 9.56 & 5.91 & 1.0 \\
${ }^{154} \mathrm{Gd}$ & 0.05 & 67.34 & 21.11 & 5.65 & 2.24 & 2.32 & 1.29 & 1.0 \\
${ }^{155} \mathrm{Gd}$ & 0.03 & 0.63 & 91.74 & 5.12 & 1.14 & 0.94 & 0.40 & 1.008 \\
${ }^{156} \mathrm{Gd}$ & 0.01 & 0.11 & 1.96 & 93.79 & 2.53 & 1.20 & 0.41 & 10.272 \\
${ }^{157} \mathrm{Gd}$ & 0.006 & 0.065 & 0.64 & 1.64 & 93.72 & 3.12 & 0.82 & 0.8 \\
${ }^{158} \mathrm{Gd}$ & 0.1 & 0.1 & 0.96 & 1.7 & 3.56 & 92.0 & 1.82 & 9.30 \\
\hline \hline
\end{tabular}

the measured $\gamma$-ray spectra and its comparison with the other available data is presented in Sec. 4 . It should be mentioned that the results for ${ }^{158} \mathrm{Gd}$ product nucleus were already published [1]. The results for other isotopes are still preliminary. A summary is given in Sec. 5.

\section{Experimental setup and measurements}

\subsection{Experimental setup}

The experiment was performed at the neutron source LANSCE [2]. The 800-MeV $\mathrm{H}^{-}$beam from the LANSCE linac is injected into the proton storage ring where it is immediately converted to $\mathrm{H}^{+}$by stripping through a thin foil. The proton pulsed beam is then extracted with a repetition rate of $20 \mathrm{~Hz}$ and strikes a tungsten spallation target. A white spectrum of neutrons with energies from subtermal up to about $1 \mathrm{MeV}$ is obtained. These neutrons are sent to flight path 14 at the Manuel Lujan Jr. Neutron Scattering Center.

The DANCE detector array is installed at $20 \mathrm{~m}$ on this flight path. It is designed for studying neutron capture cross sections on small samples [3,4]. DANCE consists of $160 \mathrm{BaF}_{2}$ scintillation crystals surrounding a sample and covering a solid angle of $\simeq 4 \pi$. A ${ }^{6} \mathrm{LiH}$ shell about 6 -cm thick is placed between the sample and the $\mathrm{BaF}_{2}$ crystals in order to reduce the scattered neutron flux striking the crystals. The remaining background due to scattered neutrons that penetrate the ${ }^{6} \mathrm{LiH}$ shell and interact with the $\mathrm{BaF}_{2}$ crystals can be subtracted, see below. Besides the $\mathrm{BaF}_{2}$ crystals, the DANCE setup includes three additional detectors that are used to monitor the neutron flux, and one detector for monitoring the external background.

Targets were made of enriched gadolinium deposited via electroplating on a beryllium foil glued to an aluminum ring. The isotopic composition of the targets is listed in Table 1 together with their average thicknesses.

\subsection{Data processing}

The DANCE acquisition system [5] is based on waveform digitization of signals from all 160 detectors using four-channel Acqiris DC265 digitizers with a sampling rate of $500 \mathrm{MS} / \mathrm{s}$ (megasamples/second). The digitizers are arranged in 14 compact PCI crates with six DC265 modules per crate. Thus one crate can handle $12 \mathrm{BaF}_{2}$ detectors with two channels - one for fast and the other for slow signal component - per detector. Each crate contains an embedded computer running under the Linux operating system, and a front-end acquisition program using the framework known as Maximum Integrated Data Acquisition System (MIDAS) [6]. The intensity of the signal from a specific crystal is collected in using a digitizer channel with suitably adjusted gains. The ratio of the fast and slow components of the signal is used for discrimination against the $\alpha$-background from natural radioactivity of $\mathrm{Ra}$ in the $\mathrm{BaF}_{2}$ crystals [4].

The energy calibration of the DANCE crystals was performed with a combination of $\gamma$-ray sources: ${ }^{137} \mathrm{Cs},{ }^{98} \mathrm{Y},{ }^{22} \mathrm{Na}$ and the intrinsic radioactivity of the detector material $\left({ }^{226} \mathrm{Ra}\right)$. The latter calibration 

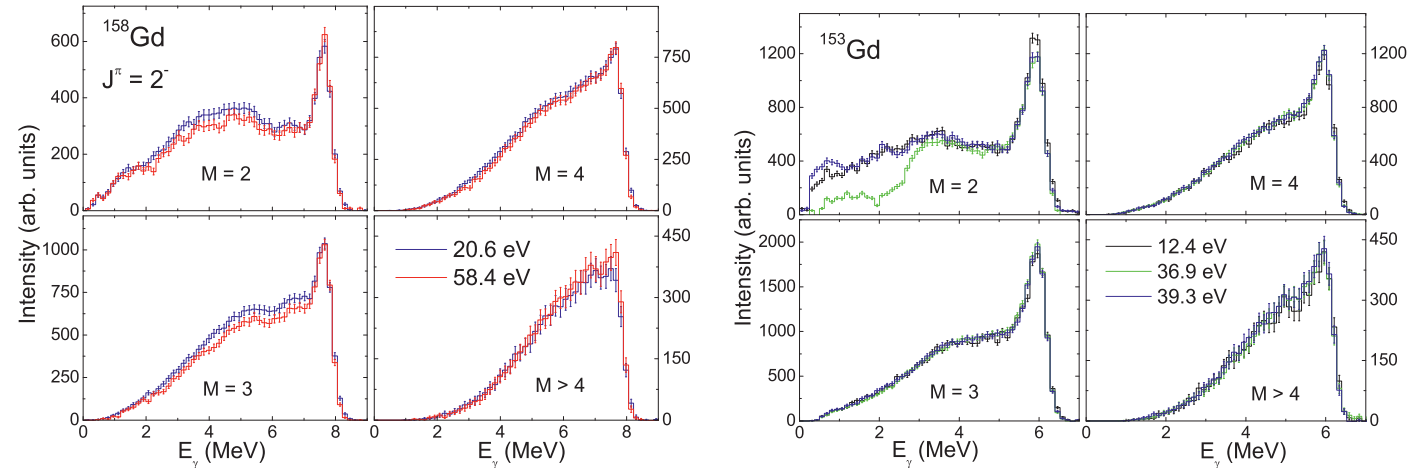

Fig. 1. Examples of sum-energy spectra obtained for resonances $J^{\pi}=2^{-}$at energies 20.6 and $58.4 \mathrm{eV}$ of the ${ }^{158} \mathrm{Gd}$ isotope (left) and the sum-energy spectra corresponding to the resonances $J^{\pi}=1 / 2^{+}$at energies $12.4,36.9$ and $39.3 \mathrm{eV}$ of the ${ }^{153} \mathrm{Gd}$ isotope (right). The cluster multiplicities of the $\gamma$ cascades, $M$, are indicated. The spectra are normalized to the intensity in the $E_{\text {total }}$ peak for multiplicities $M=2-7$.

was conducted on a run-by-run basis to provide the energy alignment of all crystals in the off-line analysis.

Typical spectra of sums of deposited $\gamma$-ray energies in crystals that fire are shown, for the case of ${ }^{157} \mathrm{Gd}$ and ${ }^{152} \mathrm{Gd}$ targets, in Fig. 1. As only $s$-wave neutron capture plays a role at low neutron energies in this mass region, we observe only $J^{\pi}=1 / 2^{+}$resonances for even Gd targets and $J^{\pi}=1^{-} \& 2^{-}$ for odd Gd targets. Each spectrum consists of (i) the "total" peak at the full energy available from the neutron capture reaction

$$
E_{\text {total }}=S_{\mathrm{n}}+E_{\mathrm{n}}
$$

where $E_{n}$ is the energy of the incoming neutron in the center of mass, and (ii) a low energy tail that corresponds to cascades for which a part of the emitted energy escaped the detector array. The shape of the spectrum at low sum energies (below about $3 \mathrm{MeV}$ ), is strongly influenced by the background from natural $\beta$ activity in the $\mathrm{BaF}_{2}$ crystals, especially for low multiplicities.

Often an emitted capture $\gamma$ ray does not deposit its full energy in one crystal. Thus the number of crystals that fire is usually higher than the true multiplicity of a capture event. Therefore all contiguous crystals that have fired during an event are combined to "clusters" and considered as the response of the detector array to a single $\gamma$-ray. The number of clusters observed in a capture event is called the "cluster" multiplicity. This multiplicity is much closer to the true multiplicity of the $\gamma$ cascade than is the "crystal" multiplicity (the total number of crystals that fire). The capture events were then sorted using gates on neutron energy and on the cluster multiplicity.

Only events within a certain range of detected sum energies $E_{\Sigma}$ (around the $E_{\text {total }}$ peak) were taken into account. During the analysis of the $\gamma$-decay in Gd isotopes a narrow, typically $1 \mathrm{MeV}$ wide, interval of $E_{\Sigma}$ was chosen for construction of multistep cascade (MSC) spectra, see below. The use of a wider interval improves statistics but leads to significant smearing of the structures seen in the spectra. Additional narrowing of the interval has virtually no impact on the spectral shape.

There is a small background contribution in the spectra that mainly originates from $\gamma$ rays following the capture of scattered neutrons in the $\mathrm{BaF}_{2}$ detectors. The size of the background can be estimated using the number of counts for $E_{\Sigma}$ above the $E_{\text {total }}$ region in the sum-energy spectra, typically at $E_{\Sigma} \approx 8-10 \mathrm{MeV}$. The background contribution from other Gd isotopes is negligible as only data from strong resonances are used. In reality, also the background from the neutron capture in crystals is small at strong resonances. 


\section{Simulation of the $\gamma$-decay of Gd isotopes}

\subsection{Simulation of spectra}

Under various assumptions about the level density and PSFs the $\gamma$ cascades following resonant neutron capture were generated using the DICEBOX algorithm [7]. The response of the DANCE detector to each generated cascade was subsequently obtained from a simulation based on the GEANT4 package. The exact geometry and chemical composition of the detector system were included in the GEANT4 simulations [8]. The resulting quantities can be compared with their experimental counterparts. In this contribution we concetrate only on the analysis of so-called MSC spectra, see Sec. 4.1, that allows us to obtain the information on the PSFs and level densities.

The DICEBOX algorithm generates a complete decay scheme of an artificial nucleus. Below some critical energy, $E_{\text {crit }}$, all of the characteristics of the decay scheme, i.e., energies, spins and parities of levels, as well as their decay properties, are taken from existing experimental data. The choice of the critical energy should be made with care to guarantee that all of the information for energies below $E_{\text {crit }}$ is complete. Above $E_{\text {crit }}$ the level system of the nucleus and its complete decay scheme are generated using an a priori chosen level density function $\rho(E, J, \pi)$ and PSFs for multipolarities $E 1, M 1$, and $E 2$. All higher multipolarities are neglected. Partial radiation width $\Gamma_{a \gamma b}$ for a transition between an initial level $a$ and a final level $b$ is given by

$$
\Gamma_{a \gamma b}=\sum_{X L} \xi_{X L}^{2} \frac{f^{(X L)} E_{\gamma}^{2 L+1}}{\rho\left(E_{a}, J_{a}, \pi_{a}\right)},
$$

where $f^{(X L)}$ stands for photon strength function for transitions of type $X$ (electric or magnetic) and multipolarity $L$, and $\xi_{X L}$ is a random number generated from a normal distribution with a zero mean and unit variance. This random number ensures that the individual widths $\Gamma_{a \gamma b}$ fluctuate according to the Porter-Thomas distribution [9]. The sum in Eq. (2) is over all allowed types and multipolarities of transitions. Internal electron conversion, which is important in transitions between the lowest excited states in Gd isotopes, is correctly treated in the DICEBOX code [7]. Hereafter the simulated system of all levels and their decay scheme is called a nuclear realization. Due to the Porter-Thomas fluctuations there exists an infinite number of nuclear realizations that differ from each other even for fixed models of PSFs and level density.

Various models of PSFs and level density can be tested with the DICEBOX code. The fluctuations involved in generating the $\gamma$ decay allow us to determine all of the uncertainties that arise when simulations are performed with the same models. Cascades starting from resonances with a given spin and parity were simulated. Typically 20 nuclear realizations, each with 100,000 cascades, were simulated for each $J^{\pi}$ of initial $s$-wave resonances. In our trial-and-error approach, by assuming various models for the PSFs and the level density, we can assess the degree of agreement of the simulated observables with the experimental data and draw conclusions about which of these models is most likely to be valid.

\subsection{Photon Strength Functions}

\subsubsection{Electric-dipole transitions}

Decay of the neutron resonances is dominated by dipole transitions. It is well known that for $\gamma$-ray energies above neutron separation energies the electric-dipole $(E 1)$ transitions play a major role. The $\mathrm{PSF}$ at these energies in axially deformed nuclei seems to be consistent with the sum of two Lorentzian terms

$$
f_{\mathrm{SLO}}^{(E 1)}\left(E_{\gamma}\right)=\frac{1}{3(\pi \hbar c)^{2}} \sum_{i=1}^{2} \frac{\sigma_{G_{i}} E_{\gamma} \Gamma_{G_{i}}^{2}}{\left(E_{\gamma}^{2}-E_{G_{i}}^{2}\right)^{2}+E_{\gamma}^{2} \Gamma_{G_{i}}^{2}} .
$$

Here $E_{G_{i}}, \Gamma_{G_{i}}$, and $\sigma_{G_{i}}$ are the parameters of the Giant Electric Dipole Resonance (GEDR) which is split into two components ( $i=1$ and 2$)$ in well-deformed nuclei. Values of these parameters for studied 


\section{$\mathrm{CNR} * 11$}

nuclei were adopted from [10]. If the parameters were not available for studied nucleus, parameters from a neighboring nucleus were adopted. This PSF shape combined with the Brink hypothesis [11], which says that the PSF shapes are independent on excitation energy, is known as the Brink-Axel or Standard Lorentzian (SLO) model.

Since the shape of the $E 1$ PSF below the neutron separation energy is not well known, additional models are employed. Usually one of two models is used. The first one was, known as KMF model, was proposed in [12] for spherical or weakly deformed nuclei, but is often also used for deformed nuclei

$$
f_{\mathrm{KMF}}^{(E 1)}\left(E_{\gamma}, T\right)=\frac{F_{K}}{3(\pi \hbar c)^{2}} \sum_{i=1}^{2} \frac{\sigma_{G_{i}} E_{G_{i}} \Gamma_{G_{i}} \Gamma\left(E_{\gamma}, T\right)}{\left(E_{\gamma}^{2}-E_{G_{i}}^{2}\right)^{2}},
$$

where the factor $F_{K}=0.7[12,13]$ and the $\gamma$-ray- and temperature-dependent width $\Gamma\left(E_{\gamma}, T\right)$ is given by

$$
\Gamma\left(E_{\gamma}, T\right)=\Gamma_{G_{i}} \frac{E_{\gamma}^{2}+4 \pi^{2} T^{2}}{E_{G_{i}}^{2}},
$$

with temperature $T=T(E) \equiv \sqrt{(E-\Delta) / a}$, the excitation energy $E$ of a final level, the pairing energy $\Delta$, and the shell-model level density parameter $a$.

A second model was proposed for spherical nuclei by Chrien [14] in order to match the behavior of the SLO model at energies near the GEDR maximum and the KMF model at very low $E_{\gamma}$. This phenomenological model was later generalized for deformed nuclei by Kopecky et al. [15] by introducing an empirical enhancement factor $k_{0}$. This model is known as the EGLO (Enhanced Generalized Lorentzian) model. In this case the expression for the PSF is

$$
f_{\mathrm{EGLO}}^{(E 1)}\left(E_{\gamma}, T\right)=\sum_{i=1}^{2} \frac{\sigma_{G_{i}} \Gamma_{G_{i}}}{3(\pi \hbar c)^{2}}\left[\frac{4 \pi^{2} F_{K} \Gamma_{G_{i}} T^{2}}{E_{G_{i}}^{5}}+\frac{E_{\gamma} \Gamma\left(E_{\gamma}, T\right)}{\left(E_{\gamma}^{2}-E_{G_{i}}^{2}\right)^{2}+E_{\gamma}^{2} \Gamma\left(E_{\gamma}, T\right)^{2}}\right]
$$

Here the $\gamma$-ray- and temperature-dependent width is given by

$$
\Gamma\left(E_{\gamma}, T\right)=\left[k_{0}+\frac{E_{\gamma}-E_{\gamma 0}}{E_{G}-E_{\gamma 0}}\left(1-k_{0}\right)\right] \Gamma_{G_{i}} \frac{E_{\gamma}^{2}+4 \pi^{2} T^{2}}{E_{G_{i}}^{2}} .
$$

The recommended value of $E_{\gamma 0}$ is $4.5 \mathrm{MeV}[13,15]$. In Ref. [13] the systematics of the parameter $k_{0}$ was adjusted to reproduce the total radiation width of neutron resonances. As the total radiation width depends on the number of levels below neutron separation energy the suggested systematics of $k_{0}$ depends on the proposed level density model. We left the parameter $k_{0}$ free to vary in our simulations. The energy dependence of the EGLO model is very similar to the KMF model for $k_{0} \approx 1.5$.

Many other models of E1 PSF can be found in the literature. RIPL-3 database [16] suggests especially the MLO family of models to be used. As the description of these models is rather complicated, the reader is referred to their detailed description in Ref. [16].

As seen from Eqs. (4) and (6) the shapes of both the KMF and EGLO models depend on temperature (or excitation energy) of the decaying nucleus and violate the strict form of the Brink hypothesis. Similar temperature dependence of E1 PSF is also a feature of the MLO family of models. The energy dependence of the PSFs predicted by these models is shown in Fig. 2 for the parametrization used for ${ }^{157} \mathrm{Gd}$ target. To keep the figure reasonably clear we show only the shape of one of the MLO models (MLO2) here.

\subsubsection{Magnetic-dipole transitions}

Magnetic dipole $(M 1)$ transitions also play an important role in the decay of highly excited nuclear states. Usually, two models are used for $M 1$ transitions. In the spin-flip (SF) resonance model it is often assumed to have a Lorentzian shape of $f_{\mathrm{SF}}^{(M 1)}\left(E_{\gamma}\right)$ with the resonance energy at about $7 \mathrm{MeV}$ and width of $4 \mathrm{MeV}$ [13], while in the single-particle model, the $f_{\mathrm{SP}}^{(M 1)}$ is a constant independent on $\gamma$-ray 


\section{EPJ Web of Conferences}
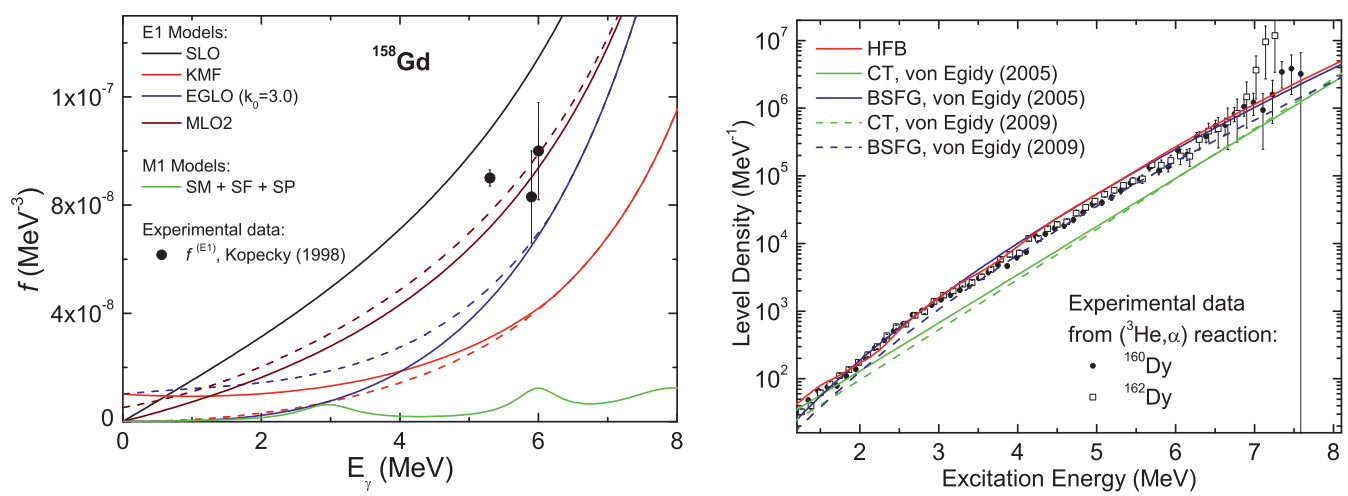

Fig. 2. Left: Some of the PSFs used in simulations of ${ }^{158} \mathrm{Gd}$. There are shown two curves for KMF, EGLO and MLO2 models which indicate the temperature dependence of these models - the lower curve corresponds to $T=0$ while the upper one to $T=\sqrt{\left(S_{\mathrm{n}}-E_{\gamma}-\Delta\right) / a}$. Experimental data adopted from Kopecky (1998) [13] for $f^{(E 1)}$ at energies 5.9, 6.0 and 5.3 $\mathrm{MeV}$ correspond to ${ }^{155,157,159} \mathrm{Gd}$, respectively. Parametrization of $M 1$ PSF corresponds to that given in Ref. [1]. Right: Level density models used in simulations of ${ }^{158} \mathrm{Gd}$. The different absolute values predicted by the various level density models originate from the different paramerization of the spin-cutoff parameter used in von Egidy (2005) [17] and von Egidy (2009) [30]. The resonance spacing of $s$-wave resonances is the same in all cases. Models of level density are compared with experimental data for even-even Dy isotopes [32].

energy. The $M 1$ strength corresponding to the spin-flip mode was measured for several rare-earth nuclei (including ${ }^{158} \mathrm{Gd}$ ) from inelastic proton scattering [18]. A double-humped structure was observed between 5 and $10 \mathrm{MeV}$ and we adopted this form of SF resonance in our simulations. Sometimes a sum of the strengths from the two models, $f_{\mathrm{SP}}^{(M 1)}$ and $f_{\mathrm{SF}}^{(M 1)}$, is used. We tested all these models and we usually adjusted the absolute value of the PSFs to obtain the ratio of $f^{(E 1)} / f^{(M 1)} \approx 7$ at about $7 \mathrm{MeV}$. This value seems to be reasonably well determined from average resonance capture experiments [19].

\subsubsection{Scissors mode}

In 1976, Hilton [20] and later Lo Iudice and Palumbo [21] and Iachello [22] predicted an isovector M1 collective vibrational mode in deformed nuclei. This mode, known as the scissors mode (SM), was experimentally observed for the ground-state transitions by Bohle et al. [23] from high-resolution electron inelastic scattering at low momentum transfer in ${ }^{156} \mathrm{Gd}$. The parameters of the mode for transitions to the ground state were intensively investigated using the $\left(\gamma, \gamma^{\prime}\right)$ reaction in rare-earth nuclei [24]. These experiments revealed substantial fragmentation of the mode and concluded that the strength of the mode (or more precisely the total $M 1$ strength in the energy range $E_{\gamma} \approx 2.5-4.0$ $\mathrm{MeV}$ ) for the ground-state transitions in even-even rare-earth nuclei is proportional to the square of the deformation [25]; for well deformed nuclei this strength reaches $B(M 1) \approx 3 \mu_{N}^{2}$ [24]. In odd nuclei the observed strength was significantly lower - about one half or one third of the strength observed in even-even nuclei. But it is believed that significant fraction of the strength in odd nuclei escaped the detection due to very high level density in the region of SM in odd nuclei [26]. The centroid of the scissors mode strength is located near $3 \mathrm{MeV}$ and is almost constant in rare-earth nuclei. In our simulations the scissors mode was represented by a single Lorentzian resonance term.

The analysis of data on two-step $\gamma$ cascades (TSC) $[27,28]$ in ${ }^{162}$ Dy revealed that the scissors mode is not only built on the ground state, but also plays a role in transitions between excited states. In other words, the scissors mode follows, at least approximately, the Brink hypothesis. This finding has been later supported by data from ${ }^{3} \mathrm{He}$-induced $\gamma$ emission [29]. 


\section{$\mathrm{CNR} * 11$}

\subsubsection{Electric-quadrupole transitions}

In addition to dipole transitions, electric quadrupole $(E 2)$ transitions might also play a role in the decay of neutron resonances. We found that $E 2$ transitions above $E_{\text {crit }}$ are not important in the interpretation of our data. We simply assumed the validity of the single-particle model $\left(f^{(E 2)}=\right.$ const. $)$ in our simulations. The strength of $f^{(E 2)}=$ const. was taken to reproduce the ratio with respect to dipole strengths at about $7 \mathrm{MeV}$ from average resonance capture data [19].

\subsection{Nuclear Level density}

Besides the PSFs a model for level density (LD) beween $E_{\text {crit }}$ and $S_{\mathrm{n}}$ is needed to simulate $\gamma$-decay of the nucleus. We mainly used the back-shifted Fermi Gas (BSFG) model [17]

$$
\rho(E, J, \pi)=f(J) f(\pi) \frac{e^{2 \sqrt{a\left(E-E_{1}\right)}}}{12 \cdot 2^{1 / 2} \sigma_{\mathrm{c}} a^{1 / 4}\left(E-E_{1}\right)^{5 / 4}},
$$

where $a$ and $E_{1}$ are adjustable parameters, and the Constant Temperature (CT) model [17]

$$
\rho(E, J, \pi)=f(J) f(\pi) \frac{1}{T} e^{\left(E-E_{0}\right) / T},
$$

where the free parameters are temperature $T$ and the energy $E_{0}$. In both models the spin probability distribution function has the form

$$
f(J)=\exp \left(\frac{-J^{2}}{2 \sigma_{\mathrm{c}}^{2}}\right)-\exp \left(\frac{-(J+1)^{2}}{2 \sigma_{\mathrm{c}}^{2}}\right) .
$$

We have also adopted the parametrization of these two models from Ref. [30] that differs from Ref. [17] mainly in form of the spin-cutoff parameter $\sigma_{\mathrm{c}}$. A strong even-odd spin staggering in the spin distribution of even-even nuclei at low excitation energies is also included in Ref. [30]. Both of these parametrizations led to virtually the same results in our analysis. No parity dependence was assumed in the BSFG and CT models, which corresponds to $f(\pi)=1 / 2$.

In addition to the closed-form models, we tested also the level density calculated within the Hartree-Fock-Bogoljubov (HFB) approach. Here, the level density is available in tabulated form as a function of energy for levels with each spin and parity [16,31]. The calculated level densities usually suffer from difficulties in reproducing the average neutron resonance spacing. In order to bring the calculations into agreement with experimental data, the HFB level density was renormalized to reproduce the resonance spacing at the neutron separation energy. After such a renormalization there is typically a very good agreement between the HFB level density and the BSFG model at energies above $E_{\text {crit }}$, see the right panel of Fig. 2.

All known levels below $E_{\text {crit }}$ are taken into account in the simulations. The level density formula is thus applied only above this energy. It is interesting to note that the adopted BSFG and HFB level density models in even-even Gd nuclei are in an excellent agreement with level densities obtained from ${ }^{3} \mathrm{He}$-induced measurements in neighboring even-even Dy isotopes [32], see Fig. 2.

\section{Properties of $\gamma$ decay}

\subsection{MSC spectra}

In order to obtain information on the properties of the $\gamma$ decay of Gd isotopes we compared experimental MSC spectra with predictions based on model simulations described in Sec. 3. The MSC spectra were constructed from capture on well-resolved strong resonances. Only $\gamma$ cascades that deposit virtually all of their energy in the DANCE detector were taken into account and sorted according to detected cluster multiplicity. Multiplicities $M=2-7$ were used in the following analysis. In order 

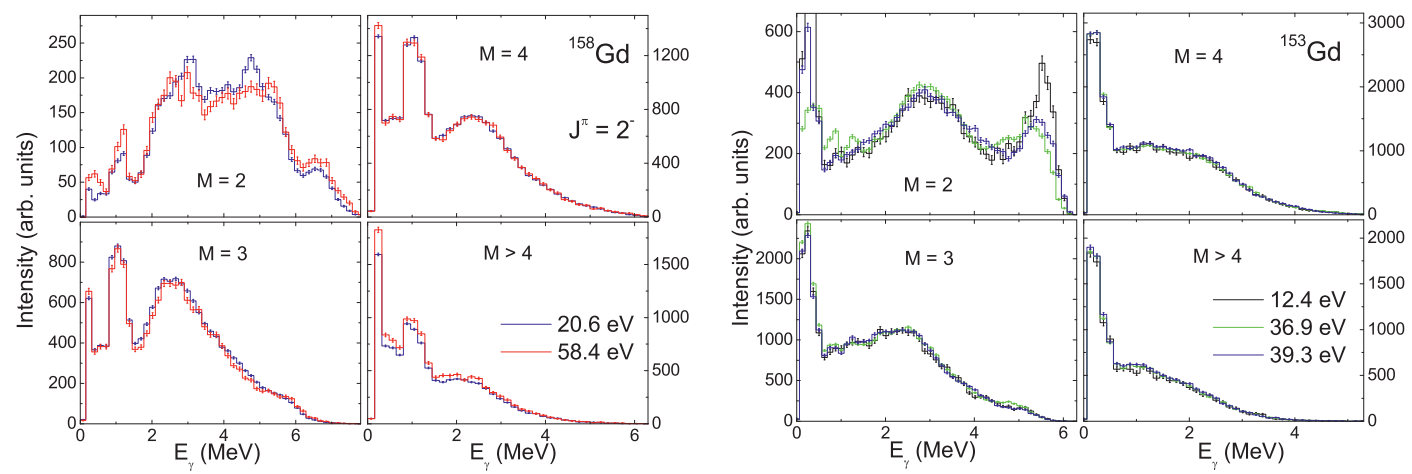

Fig. 3. Experimental MSC spectra obtained for selected $J^{\pi}=2^{-}$neutron resonances of ${ }^{158} \mathrm{Gd}$ (left) and selected $J^{\pi}=1 / 2^{+}$neutron resonances of ${ }^{153} \mathrm{Gd}$ (right) isotopes.

to minimize statistical uncertainties as well as uncertainties from simulations, the spectra were binned into coarse bins with a width of $150 \mathrm{keV}$. As already noted, the background contribution to the MSC spectra is very small for strong resonances.

A large number (several hundreds) of model combinations (parametrizations) of PSFs and level densities was tested in simulations and compared with the experimental MSC spectra. It is very difficult to quantify the agreement between simulations and experimental spectra as individual bins in the MSC spectra are mutually correlated in a complicated way. As a consequence, the degree of agreement was only checked visually.

For all multiplicities, only one normalization parameter is needed for comparison of experimental and simulated MSC spectra. We normalized spectra to the same number of counts in the $E_{\text {total }}$ peak, which includes almost all multiplicities. As already noted, in practice we considered multiplicities $M=2-7$. The experimental MSC spectra from resonances with the same spin are similar but not identical, due to Porter-Thomas fluctuations of the primary transitions. This is illustrated for ${ }^{158} \mathrm{Gd}$ and ${ }^{153} \mathrm{Gd}$ isotopes in Fig. 3.

For the same reason the simulated MSC spectra for different nuclear realizations obtained with the same model of PSFs and level density are not identical. To characterize uncertainties due to PorterThomas fluctuations the predicted MSC spectra are plotted as a gray band. Each such band has a width of two sigma (the average \pm one sigma) and was obtained from analysis of 20 independent nuclear realizations. The size of fluctuations among the MSC spectra for different resonances seems to be well reproduced by the simulations. The spectra from different nuclear realizations are almost identical for higher multiplicities $(M \geq 4)$, while some differences are predicted for lower multiplicities, especially for $M=2$.

\subsection{Comparison of experimental and simulated spectra}

Models that do not include a resonance structure near $3 \mathrm{MeV}$ in a PSF are unable to reproduce the humps situated at this energy observed in the $M=2-4$ MSC spectra in none of Gd isotopes. Typical examples of simulated MSC spectra with no resonance structure are shown in Fig. 4.

Simulations also show that the resonance structure cannot be of $E 1$ character, see Fig. 5. On the other hand, a resonance structure near $3 \mathrm{MeV}$ in the $M 1$ or E2 PSF is able to describe experimental MSC spectra. We assume in the following that such a resonance structure is in the M1 PSF, i.e., it is the scissors mode (SM).

To reach a reasonable agreement with the experimental data the SM must be postulated also on excited states, i.e. it must follow - at least approximately - the Brink hypothesis. An example of simulations with the $M 1$ which violates the Brink hypothesis is shown in Fig. 6.

We found that the MSC spectra are rather sensitive to the energy of the scissors mode, $E_{\mathrm{SM}}$. The dependence of the MSC spectra on the resonance damping width, $\Gamma_{\mathrm{SM}}$, and on the total strength of 

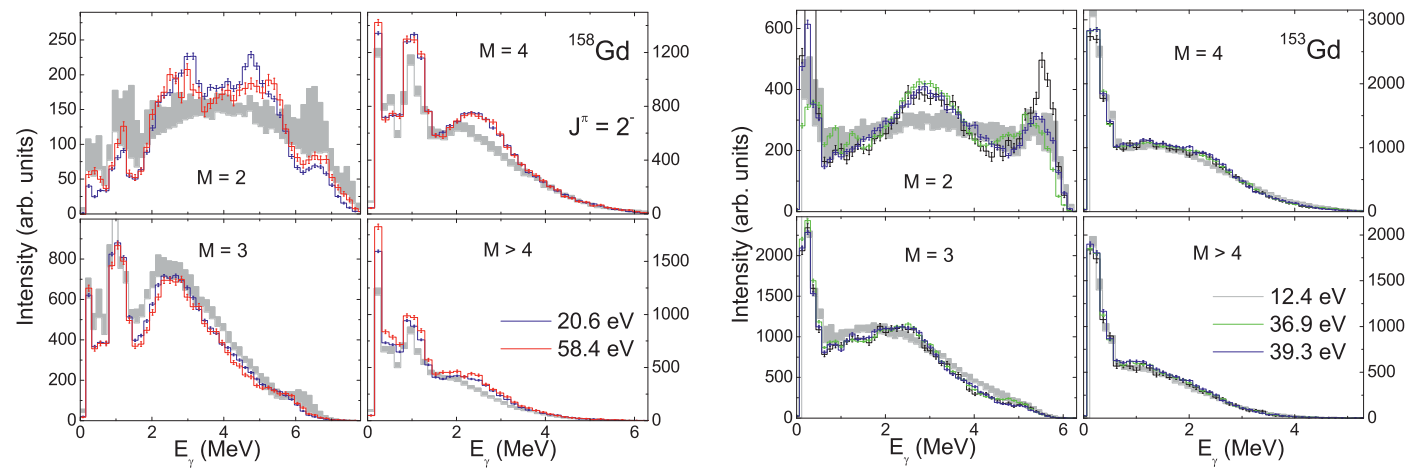

Fig. 4. Comparison of experimental MSC spectra with the simulations (grey band) that assume the entire absence of the SM in PSFs. The model combination of $f_{\mathrm{KMF}}^{(E 1)}, f_{\mathrm{SP}}^{(M 1)}=1 \times 10^{-9} \mathrm{MeV}^{-3}+f_{\mathrm{SF}}^{(M 1)}, f_{\mathrm{SP}}^{(E 2)}=5 \times 10^{-11} \mathrm{MeV}^{-5}$ PSFs with BSFG model [17] of LD is compared with the experimental data obtained for the selected $J^{\pi}=2^{-}$ neutron resonances of ${ }^{158} \mathrm{Gd}$ nucleus (left) and selected $J^{\pi}=1 / 2^{+}$neutron resonances of ${ }^{153} \mathrm{Gd}$ nucleus (right).
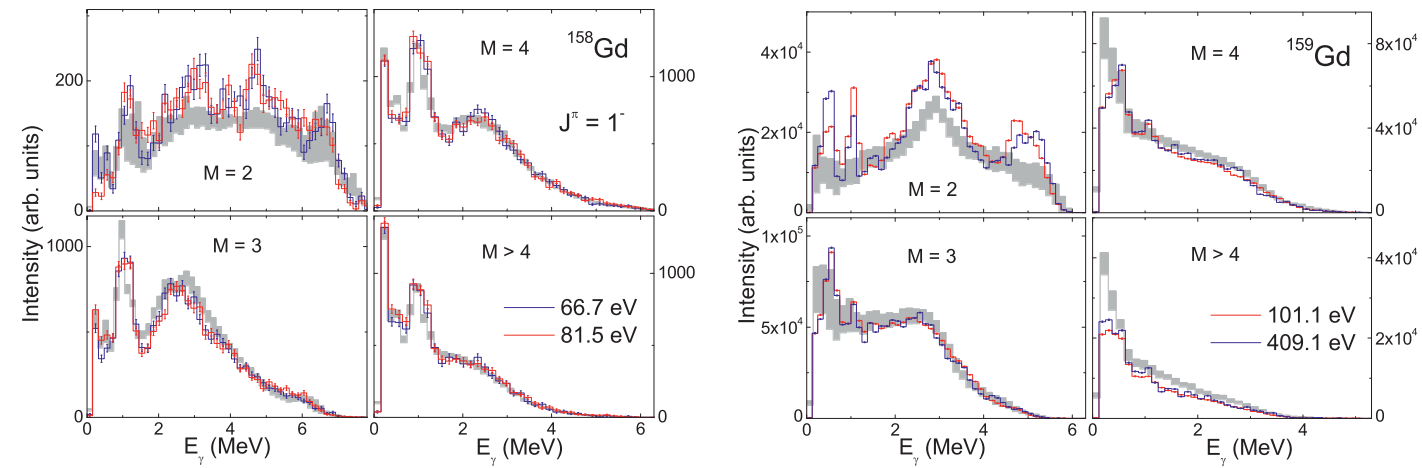

Fig. 5. Comparison of experimental MSC spectra with the simulations (grey band) which assume the presence of the Lorentz-like resonance structure at $3 \mathrm{MeV}$ in $E 1$ instead of $M 1 \mathrm{PSF}$ is shown for ${ }^{158} \mathrm{Gd}$ (left) and ${ }^{159} \mathrm{Gd}$ (right) isotopes.

the scissors mode, which is given by the product $\sigma_{\mathrm{SM}} . \Gamma_{\mathrm{SM}}$, is much weaker. The position of the mode must be very close to $3 \mathrm{MeV}$. We estimate that in all Gd isotopes, except the ${ }^{155} \mathrm{Gd}$ nucleus, it cannot be lower than about $2.8 \mathrm{MeV}$ or higher than about $3.1 \mathrm{MeV}$. In case of ${ }^{155} \mathrm{Gd}$ very preliminary results indicate a position between 2.6 and $2.8 \mathrm{MeV}$. If the resonance energy is outside this range the shapes of the bumps in the MSC spectra for $M=2-4$ are not reproduced. This restriction on the $E_{\mathrm{SM}}$ - at least in even-even nuclei - leads, for example, to a disagreement between the present experimental data and simulations with the PSF deduced from $\left({ }^{3} \mathrm{He}, \alpha\right)$ reaction in neighboring even-even Dy nuclei, see [1]. Rather surprisingly, predictions based on very different damping widths yielded similar results. The spectra seem to allow any value between $\Gamma_{\mathrm{SM}}=0.7$ and $1.3 \mathrm{MeV}$.

In majority of $\mathrm{Gd}$ isotopes we were unable to reach a reasonable agreement between the simulated and experimental spectra for any model combination incorporating the SLO model for the E1 PSF. On the other hand, a reasonably good agreement is achieved with the KMF, as well as with the EGLO model (with the dimensionless constant $k_{0}$ adjusted to 1.5-3.5), in combination with a "composite" model of the M1 PSF: $f^{(M 1)}=f_{\mathrm{SM}}^{(M 1)}+f_{\mathrm{SF}}^{(M 1)}+f_{\mathrm{SP}}^{(M 1)}$. In even-even product nuclei the simulated MSC spectra are virtually insensitive to $\sigma_{\mathrm{SM}}$ within the range of $0.07-0.25 \mathrm{mb}$ and to $f_{\mathrm{SP}}^{(M 1)}$ within the range $(1-2.5) \times 10^{-9} \mathrm{MeV}^{-3}$. In the preliminary results obtained for odd product nuclei there seems to be 

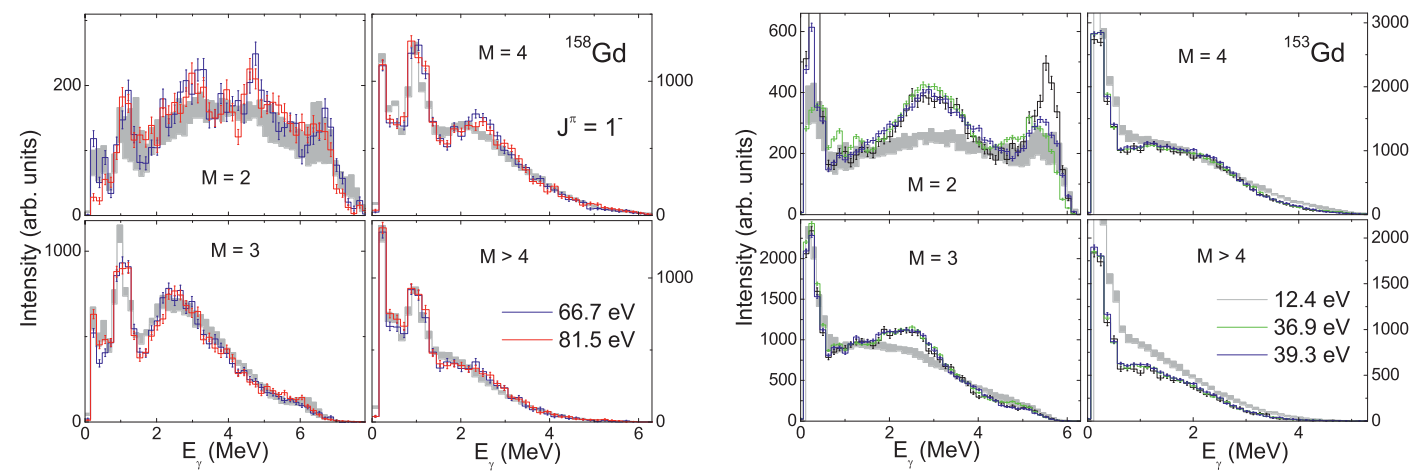

Fig. 6. Comparison of experimental MSC spectra with the calculations assuming the presence of the SM only for the ground state transitions. In case of ${ }^{158} \mathrm{Gd}$ nucleus (left) the SM parametrization is $E_{\mathrm{SM}}=3.0 \mathrm{MeV}$, $\Gamma_{\mathrm{SM}}=1.0 \mathrm{MeV}$ and $\sigma_{\mathrm{SM}}=0.2 \mathrm{mb}$, while for the ${ }^{153} \mathrm{Gd}$ nucleus (right) it is $E_{\mathrm{SM}}=2.9 \mathrm{MeV}, \Gamma_{\mathrm{SM}}=1.0 \mathrm{MeV}$ and $\sigma_{\mathrm{SM}}=0.2 \mathrm{mb}$. In addition to the SM there are also $f_{\mathrm{SP}}^{(M 1)}$ and $f_{\mathrm{SF}}^{(M 1)}$ present in $M 1$ PSF with the same parameters as in Fig. 4. Models used for E1, E2 and LD are the same as in Fig. 4.
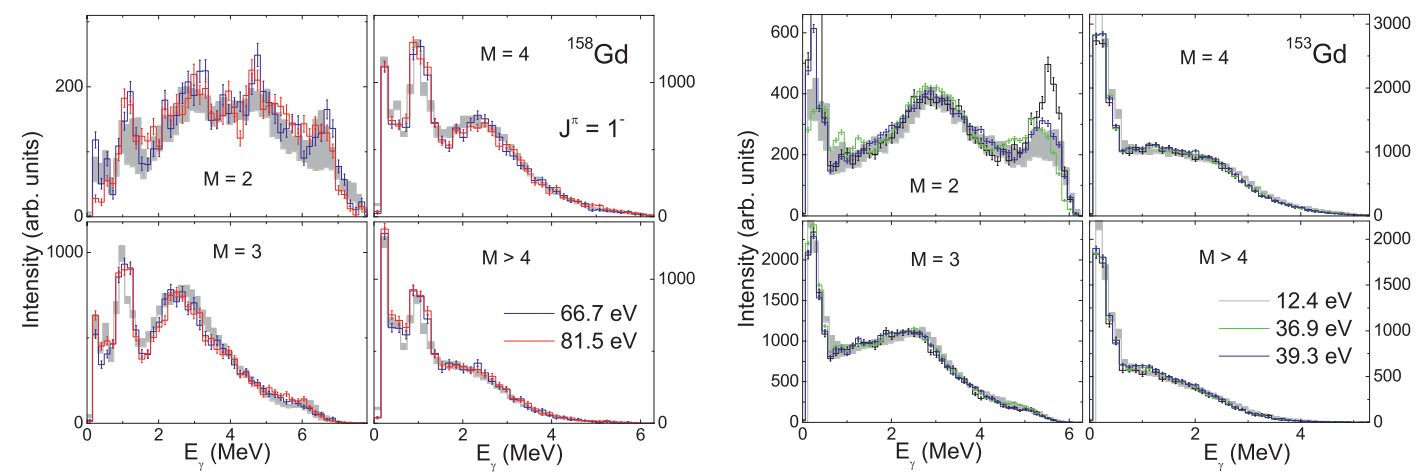

Fig. 7. Calculated MSC spectra compared with the experimental data obtained for selected $J^{\pi}=1^{-}$neutron resonances of ${ }^{158} \mathrm{Gd}$ (left) and $J^{\pi}=1 / 2^{+}$neutron resonances of ${ }^{153} \mathrm{Gd}$ (right) nuclei. The SM parametrization is the same as in Fig. 6, but it is assumed here that the SM is built on all the excited levels. There are also $f_{\mathrm{SP}}^{(M 1)}$ and $f_{\mathrm{SF}}^{(M 1)}$ present in $M 1 \mathrm{PSF}$ with the same parameters as in Fig. 4. Models used for E1, E2 and LD are the same as in Fig. 4.

a systematic trend - the heavier nucleus, the higher $\sigma_{\mathrm{SM}}$ running from about $\sigma_{\mathrm{SM}}=0.2 \mathrm{mb}$ in ${ }^{153} \mathrm{Gd}$ to about $\sigma_{\mathrm{SM}}=0.6 \mathrm{mb}$ in ${ }^{159} \mathrm{Gd}$, see Figs. 7 and 8 .

Omitting the $f_{\mathrm{SP}}^{(M 1)}$ part of $M 1$ PSF seems to worsen the agreement in all tested nuclei. There is a very slight sensitivity of the shapes of simulated spectra to the parameters of $f_{\mathrm{SF}}^{(M 1)}$ and to the E2 strength. We have obtained satisfactory agreement between the MSC experimental spectra and the simulations only using the BSFG model $[17,30]$ for level density while the calculations with the CT formula $[17,30]$ didn't lead to the reasonable agreement in any studied isotopes.

We should stress that within an enormous functional space the trial-and-error method adopted in our analysis does not guarantee that we find the models (or combinations of parameters) that lead to the best possible agreement between simulated and experimental MSC spectra.

With the exception of the SLO model and the model based on data from ${ }^{3} \mathrm{He}$-induced reactions, we did not test models where the E1 PSF does not depend on temperature (in other words models that fully obey the Brink hypothesis). As a consequence, we are unable to decide whether we need 

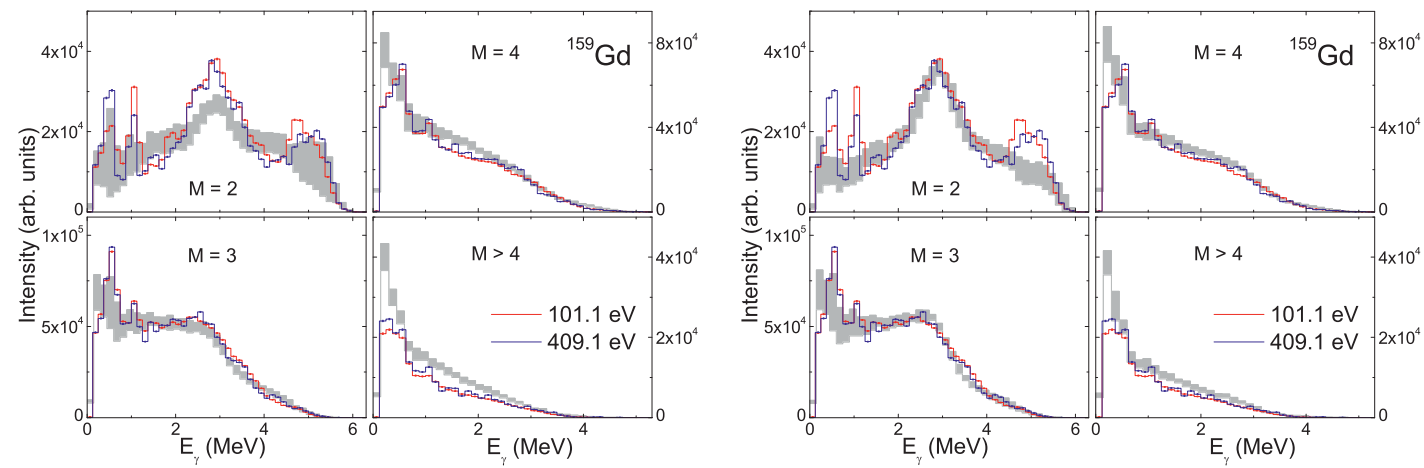

Fig. 8. Comparison of the experimental $\gamma$-ray spectra obtained for the nucleus ${ }^{159} \mathrm{Gd}$ with the simulations assuming the SM strength $\sigma_{\mathrm{SM}}=0.2 \mathrm{mb}$ (left) and $\sigma_{\mathrm{SM}}=0.5 \mathrm{mb}$ (right). The position $E_{\mathrm{SM}}=3.0 \mathrm{MeV}$ and the width $\Gamma_{\mathrm{SM}}=1.0 \mathrm{MeV}$ of SM are the same in both figures. The $f_{\mathrm{SP}}^{(M 1)}$ and $f_{\mathrm{SF}}^{(M 1)}$ PSFs are also present in $M 1$ PSF with the same parameters as in Fig. 4. Models used for E1, E2 and LD are the same as in Fig. 4.

a temperature-dependent $E 1 \mathrm{PSF}$ in order to reproduce the MSC spectra. We can only conclude that the MSC spectra are consistent with predictions of "temperature-dependent" KMF or EGLO models.

\subsection{Comparison with other data}

There are several other relevant measurements that provide information on the PSFs below the neutron separation energy for $A \sim 150-160$ nuclei. Data on ground-state transitions from $\left(\gamma, \gamma^{\prime}\right)$ measurements $[24,33]$, that are available for many even-even and few odd nuclei, and data from ${ }^{3} \mathrm{He}$-induced reactions [29] in neighboring nuclei were already mentioned. In addition, there also exist data from the $(n, \gamma)$ reaction. Specifically, (i) values of the PSFs were obtained from the intensities of primary transitions from resonance neutron capture in several odd nuclei [13], see also Fig. 2, (ii) two-step $\gamma$ cascades following thermal neutron capture in ${ }^{162}$ Dy were measured [28], and (iii) information on the total radiation widths of neutron resonances is available for all stable isotopes [34].

As already mentioned in Sec 3.2.3, data from $\left(\gamma, \gamma^{\prime}\right)$ experiments yield the total reduced $M 1$ strength for transitions to the ground state of $B(M 1) \approx 3 \mu_{N}^{2}$ for $E_{\gamma}$ between 2.7 and $3.7 \mathrm{MeV}$ in well deformed even-even nuclei. In our case the total reduced strength of the SM in a comparable energy interval obtained for the ${ }^{156} \mathrm{Gd}$ and ${ }^{158} \mathrm{Gd}$ isotopes is $B(M 1) \approx 1.5 \mu_{\mathrm{N}}^{2}$. It means that for both eveneven $\mathrm{Gd}$ isotopes our data require significantly smaller $B(M 1)$. In fact, we should not compare only the strength of the scissors mode, but rather the sum of all $M 1$ contributions, $f^{(M 1)}=f_{\mathrm{SM}}^{(M 1)}+f_{\mathrm{SP}}^{(M 1)}+f_{\mathrm{SF}}^{(M 1)}$, with experimental data. The $f_{\mathrm{SF}}^{(M 1)}$ contribution to the given energy interval is very weak, $<0.2 \mu_{N}^{2}$. The contribution of $f_{\mathrm{SP}}^{(M 1)}$ is slightly higher, but still $\leq 0.5 \mu_{N}^{2}$. In any case, the $B(M 1)$ in even-even Gd nuclei needed for reproduction of our data is at most about $2 \mu_{N}^{2}$. The observed difference in the strengths from $\left(\gamma, \gamma^{\prime}\right)$ and our experiments suggests that the parameters of the scissors mode for ground-state transitions may differ from the corresponding parameters for excited levels. Such a situation would not be at strong variance with our data. For instance in the case of ${ }^{158} \mathrm{Gd}$ simulations with the SM that gives $B(M 1) \sim 3 \mu_{N}^{2}$ for the ground state transitions but $B(M 1) \sim 1.1 \mu_{N}^{2}$ for transitions between all excited states showed negligible difference with respect to simulations with the SM giving $B(M 1) \sim 1.1 \mu_{N}^{2}$ independently of the final level [1].

The strength in odd Gd isotopes (still preliminary) seems to increase with the increasing mass number. For the lightest Gd isotopes it is very similar to the strength in even-even Gd nuclei, while the value about 2.5 or 3 times higher is observed for ${ }^{157} \mathrm{Gd}$ and ${ }^{159} \mathrm{Gd}$ isotopes, see Fig. 8. An increase in the strength of the SM with $A$ in odd Gd chain might be expected as a deformation of these isotopes increases too. On the other hand, the change in deformation is only weak [16] and the dependence 


\section{EPJ Web of Conferences}

of the SM strength does not seem to follow the square of deformation as it is suggested from NRF experiments in even-even nuclei.

\section{Summary}

Measurement of $\gamma$-ray spectra from resonances in 6 stable Gd isotopes was performed with an isotopically enriched targets at the DANCE detector array at LANSCE. The multistep cascade $\gamma$-ray spectra for different multiplicities were used to test the validity of various PSF models. For the $E 1$ PSF we found that, at least at low $\gamma$-ray energies, a reasonable description was obtained with the KMF or $\operatorname{EGLO}\left(k_{0}=1.5-3.5\right)$ models.

Our analysis indicates that a resonance-like structure at $E_{\gamma} \approx 3 \mathrm{MeV}$ in a PSF in all Gd isotopes is required. The structure is not only a property of the ground-state transitions but must also be present in the decay between excited levels. We identify this structure with the scissors mode as it cannot be in the $E 1$ PSF. It is relatively wide, we estimate its width to be $0.7-1.3 \mathrm{MeV}$. In addition to the scissors mode additional "smooth" $M 1$ strength seems to be needed to reproduce our data.

The strength of the mode from our data on even-even Gd nuclei is significantly smaller than that of the ground-state transitions from $\left(\gamma, \gamma^{\prime}\right)$ experiments, what may suggest that the properties of the mode might be different for the ground-state transitions and for transitions between excited levels. One cannot completely exclude also a systematic decrease of the scissors mode strength with the excitation energy of the levels on which this mode is based. The difference between the strength of the scissors mode in heavier odd and even-even Gd nuclei could indicate that the odd nucleon plays a very important role in the systematic of the scissors mode strength.

\section{ACKNOWLEDGEMENTS}

This work was supported in part by the U. S. Department of Energy Grants No. DE-FG52-09NA29460 and No. DE-FG02-97-ER41042. This work benefited from the use of the LANSCE accelerator and was performed under the auspices of the U. S. Department of Energy at Los Alamos National Laboratory by the Los Alamos National Security, LLC under Contract No. DE-AC52-06NA25396 and at the Lawrence Livermore National Laboratory by the Lawrence Livermore National Security, LLC under Contract No. DE-AC52-07NA27344. It was also supported by the research plans MSM 0021620859, and INGO LA08015 of the Ministry of Education of the Czech Republic, and grant SVV-2011-263309 of the Charles University in Prague.

\section{References}

1. A. Chyzh et al., Phys. Rev. C 84, 014306 (2011).

2. P. W. Lisowski et al., Nucl. Sci. Eng. 106, 208 (1990).

3. M. Heil, R. Reifarth, M. M. Fowler, R. C. Haight, F. Käppeler, R. S. Rundberg, E. H. Seabury, J. L. Ullmann and K. Wisshak, Nucl. Instrum. Methods Phys. Res. A 459, 229 (2001).

4. R. Reifarth et al., Nucl. Instrum. Methods Phys. Res. A 531, 530 (2004).

5. J. M. Wouters et al., IEEE Transactions on Nuclear Science 53, 880 (2006).

6. S. Ritt and P.-A. Amaudruz, MIDAS - Maximum Integrated Data Acquisition System, http://midas.psi.ch.

7. F. Bečvář, Nucl. Instrum. Methods Phys. Res. A 417, 434 (1998).

8. M. Jandel et al., Nucl. Instrum. Methods Phys. Res. B 261, 1117 (2007).

9. C. E. Porter and R. G. Thomas, Phys. Rev. 104, 483 (1956).

10. S. S. Dietrich and B. L. Berman, At. Data and Nucl. Data Tables 38, 199 (1988).

11. D. M. Brink, Ph.D. thesis, Oxford University, 1955.

12. S. G. Kadmenskij, V. P. Markushev, and V. I. Furman, Sov. J. Nucl. Phys. 37, 165 (1983). 


\section{$\mathrm{CNR} * 11$}

13. J. Kopecky, in Handbook for Calculations of Nuclear Reaction Data, Report No. IAEATECDOC-1034 (IAEA, Vienna, 1998), p. 97.

14. R. E. Chrien, in Proc. of the Vth International School on Neutron Physics, Alushta, Dubna 1987, ed. by B.B. Kolesova and V.R. Sarantseva (Dubna Report No. D3, 4, 17-86-747, 1987).

15. J. Kopecky, M. Uhl and R. E. Chrien, Phys. Rev. C 47, 312 (1993).

16. R. Capote et al., Nucl. Data Sheets 1103107 (2009).

17. T. von Egidy and D. Bucurescu, Phys. Rev. C 72044311 (2005).

18. D. Frekers et al., Phys. Lett. B244, 178(1990).

19. L. M. Bollinger and G. E. Thomas, Phys. Rev. C 2, 1951 (1970).

20. R. R. Hilton, in Proceedings of the International Conference on Nuclear Structure, Dubna, 1976 (unpublished).

21. N. Lo Iudice and F. Palumbo, Phys. Rev. Lett. 41, 1532 (1978)

22. F. Iachello, Nucl. Phys. A358, 89c (1981).

23. D. Bohle et al., Phys. Lett. B137 (1984) 27.

24. U. Kneissl, H. H. Pitz and A. Zilges, Prog. Part. Nucl. Phys. 37 (1996) 349.

25. W. Ziegler, C. Rangacharyulu, A. Richter, and C. Spieler, Phys. Rev. Lett. 65, 2515 (1990).

26. A. Nord et al., Phys. Rev. C 67, 034307 (2003).

27. F. Bečvář, P. Cejnar, J. Honzátko, K. Konečný, I. Tomandl and R. E. Chrien, Phys. Rev. C 521278 (1995).

28. M. Krtička, F. Bečvář, J. Honzátko, I. Tomandl, M. Heil, F. Käppeler, R. Reifarth, F. Voss, and K. Wisshak, Phys. Rev. Lett. 92, 172501 (2004).

29. A. Schiller, A. Voinov, E. Algin, J. A. Becker, L. A. Bernstein, P. E. Garrett, M. Guttormsen, R. O. Nelson, J. Rekstad, and S. Siem, Phys. Lett. B633, 225 (2006).

30. T. von Egidy and D. Bucurescu, Phys. Rev. C 80054310 (2009).

31. S. Goriely, Nucl. Phys. A605 (1996) 28.

32. M. Guttormsen et al., Phys. Rev. C 68, 064306 (2003).

33. H. H. Pitz, U. E. P. Berg, R. D. Heil, U. Kneissl, R. Stock, C. Wesselborg, and P. von Brentano, Nucl. Phys. A492, 411 (1989).

34. S. F. Mughabghab, Atlas of Neutron Resonances, (Elsevier, Amsterdam), 2006. 